PREPARED FOR THE U.S. DEPARTMENT OF ENERGY, UNDER CONTRACT DE-AC02-76CH03073

PPPL-3750

PPPL-3750

UC-70

Measurement of the Transverse Spitzer Resistivity during Collisional Magnetic Reconnection

by

F. Trintchouk, M. Yamada, H. Ji, R.M. Kulsrud, and T.A. Carter

September 2002

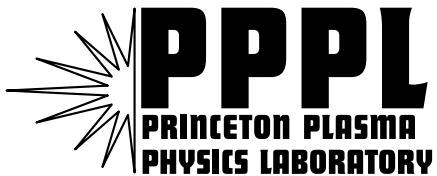

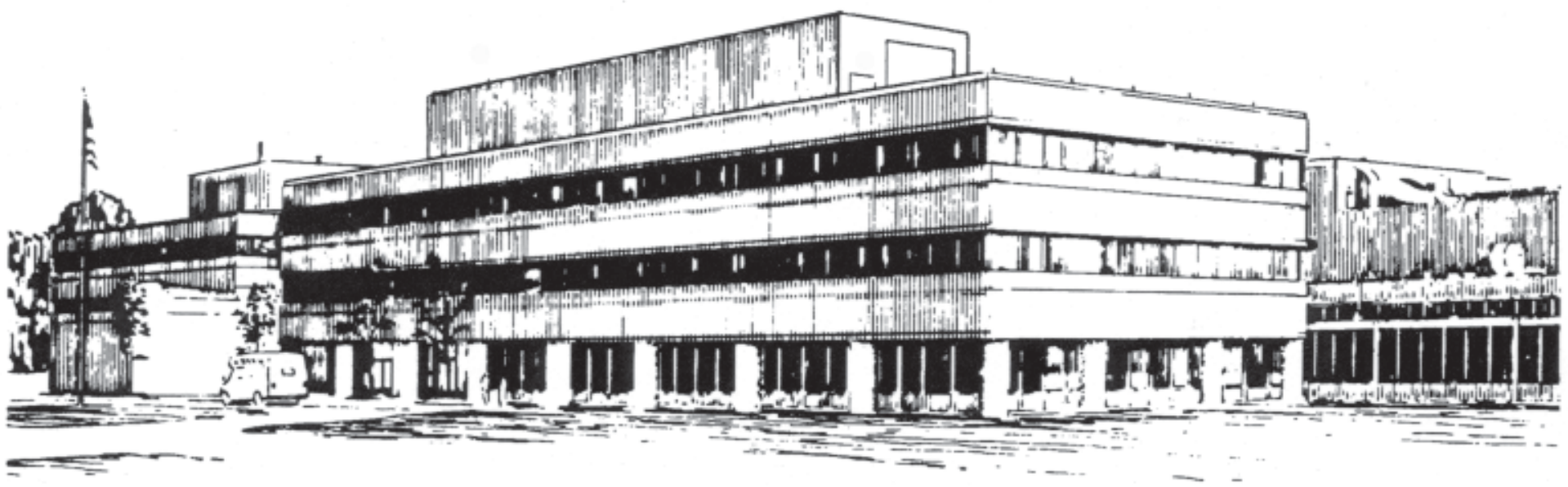

PRINCETON PLASMA PHYSICS LABORATORY PRINCETON UNIVERSITY, PRINCETON, NEW JERSEY 


\section{PPPL Reports Disclaimer}

This report was prepared as an account of work sponsored by an agency of the United States Government. Neither the United States Government nor any agency thereof, nor any of their employees, makes any warranty, express or implied, or assumes any legal liability or responsibility for the accuracy, completeness, or usefulness of any information, apparatus, product, or process disclosed, or represents that its use would not infringe privately owned rights. Reference herein to any specific commercial product, process, or service by trade name, trademark, manufacturer, or otherwise, does not necessarily constitute or imply its endorsement, recommendation, or favoring by the United States Government or any agency thereof. The views and opinions of authors expressed herein do not necessarily state or reflect those of the United States Government or any agency thereof.

\section{Availability}

This report is posted on the U.S. Department of Energy's Princeton Plasma Physics Laboratory Publications and Reports web site in Fiscal Year 2002. The home page for PPPL Reports and Publications is: http://www.pppl.gov/pub_report/

DOE and DOE Contractors can obtain copies of this report from:

U.S. Department of Energy

Office of Scientific and Technical Information

DOE Technical Information Services (DTIS)

P.O. Box 62

Oak Ridge, TN 37831

Telephone: (865) 576-8401

Fax: (865) 576-5728

Email: reports@adonis.osti.gov

This report is available to the general public from:

National Technical Information Service

U.S. Department of Commerce

5285 Port Royal Road

Springfield, VA 22161

Telephone: 1-800-553-6847 or

(703) 605-6000

Fax: (703) 321-8547

Internet: http://www.ntis.gov/ordering.htm 


\title{
Measurement of the transverse Spitzer resistivity during collisional magnetic reconnection
}

\author{
F. Trintchouk*, M. Yamada, H. Ji, R. M. Kulsrud and T. A. Carter \\ Princeton Plasma Physics Laboratory, P.O. Box 451, Princeton, New Jersey 08543
}

(September 15, 2002)

\begin{abstract}
Measurement of the transverse resistivity was carried out in a reconnecting current sheet where the mean free path for the Coulomb collision is smaller than the thickness of the sheet. In a collisional neutral sheet without a guide field, the transverse resistivity is directly related to the reconnection rate. A remarkable agreement is found between the measured resistivity and the classical value derived by L. Spitzer. In his calculation the transverse resistivity for the electrons is higher than the parallel resistivity by a factor of 1.96 . The measured values have verified this theory to within $30 \%$ errors.
\end{abstract}

52.25.Fi, 52.25.Xz

Typeset using REVTEX 
Coulomb collisions among charged particle species were historically the first mechanism of transport in plasmas to be described by a quantitative theory [1]. In magnetically confined plasma devices this "classical" mechanism is often strongly modified by particle orbit effects, or is completely dominated by turbulent transport. Nevertheless, the classical value of electrical resistivity, among other transport coefficients, is universally used as an important reference value and the lower bound whenever transport or dissipation phenomena are discussed.

For plasmas where Coulomb collisions dominate all other dissipation processes, including wave and turbulence effects the resistivity is determined by the collisional drag on electrons moving against the background of ions. If a strong magnetic field is applied perpendicular to the electric field direction, the current is not due to direct acceleration of electrons by the electric field but is diamagnetic in origin. The transverse or cross-field resistivity was calculated by L. Spitzer [1] as the rate of momentum transfer from electrons to ions through collisions:

$$
\eta_{\perp}=1.03 \times 10^{-4} T_{e}^{-3 / 2} Z \ln \Lambda(\mathrm{Ohm} \cdot \mathrm{m})
$$

where the electron temperature $T_{e}$ is in electron-volts and $\ln \Lambda$ is the Coulomb logarithm. This resistivity arises from $e-i$ collisions as the electrons drift with respect to the ions and is determined by the electron velocity. What drives the current or which species carries it in the laboratory frame is immaterial to Spitzer's definition. This transverse resistivity is approximately twice as large as the resistivity without the presence of the magnetic field since the transverse electron distribution function is not as distorted as in unmagnetized plasma where the current is carried by energetic electrons experiencing less frequent collisions. We report the first quantitative measurement of the Spitzer's transverse resistivity within the accuracy of $30 \%$, in a highly collisional magnetically reconnecting plasma.

The classical parallel resistivity is equal to that of an unmagnetized plasma. Its value has been verified experimentally with precision in cylindrical Q-machines by driving current on the axis of symmetry [2], and later in tokamaks [3]. To the authors' knowledge no 
measurements of comparable quality have been reported for the transverse resistivity. The difficulty of such measurement is due to the $E \times B$ drift resulting from the application of the transverse electric field. In a typical plasma that incorporates the unrestrained $E \times B$ flow the two terms on the left hand side of Ohm's law $\boldsymbol{E}+\boldsymbol{v} \times \boldsymbol{B}=\eta j$ very nearly cancel each other. Attempting to experimentally evaluate Ohm's law fails to produce a reliable estimate of $\eta$ since it involves subtracting two nearly identical numbers. One way to make the measurement feasible is to suppress the cross-field flow, which can be achieved by designing an equilibrium with a magnetic null between regions with oppositely directed fields. Plasma volumes with oppositely directed fields are brought into contact and the $E \times B$ motions are stagnated against each other. This situation occurs in the field-reversed configuration (FRC). The flux confinement time observed in FRCs created in $\theta$-pinch machines has so far provided the only experimental estimates of $\eta_{\perp}$. The transport in these devices has been found to be highly anomalous [4], with the resistivities implied by the flux decay generally exceeding the classical values by a factor from several times to several orders of magnitude. Various MHD and micro-instabilities were found to correlate with the anomaly factor [5]. These instabilities, rather then collisions are thought to be the dominant dissipation mechanism in these experiments.

This letter presents resistivity measurements in the neutral sheet of Magnetic Reconnection Experiment (MRX), where an electric field is applied perpendicular to the reconnecting magnetic field. Oppositely directed magnetic field lines merge through the neutral sheet, as a pressure gradient is created perpendicularly to both $E$ and $B$ (as in FRCs) and a neutral sheet current is induced. If the plasma were perfectly conductive, this sheet current would prevent the reconnection of the field lines. If the plasma is collisional, a resistance force is created against the electric field, the sheet current dissipates and reconnection takes place. We measured the Spitzer's transverse resistivity in a highly collisional region of a reconnecting plasma where the mean free path for Coulomb collisions is smaller than the neutral sheet thickness.

Fig. 1 (a) shows a poloidal cross-section view of the MRX vacuum vessel. The plasma 
is formed by inductive discharge driven by two sets of coils contained in two toroidal flux cores [6]. The plasma assumes an annular shape surrounding the flux cores. The reconnection driving forces are controlled by the rate at which a toroidal current is induced in the annular plasmas. Not shown are equilibrium field coils. The configuration allows the study of reconnection under a variety of external driving conditions. The present experiments deal with antiparallel reconnection, for which the toroidal guide field is absent. Without a guide field, the experiment is strictly two-dimensional so the transverse resistivity is directly related to the measured reconnection rate. Following the formation of the plasma, the initial force-free magnetic field configuration (Fig. 1 (a)) has an x-point on the midplane of the device. To trigger the so-called "pull" reconnection depicted in Fig. 1 (b), the externally applied poloidal field is ramped down. As a result, the poloidal flux is pulled radially toward the x-point, and a characteristic toroidal current sheet forms. The typical conditions are as follows: density $0.1-1.5 \times 10^{14} \mathrm{~cm}^{-3}$, electron temperature $3-20 \mathrm{eV}$, and $B<0.5 \mathrm{kG}$.

The Spitzer model used to obtain Eq. (1) relies on the following assumptions: (1) steady state: $\partial / \partial t=0,(2)$ uniform magnetic field, (3) the electrons are magnetized and (4) collisional: $\rho_{e} \ll \lambda_{m f p} \ll \delta$, (5) the electric field is small enough so that the deviation of the electron distribution function from Maxwellian is small: $E \lambda_{m f p}<T_{e}$, (6) collisions with neutral species are negligible. Here $\lambda_{m f p}$ is the electron mean free path due to Coulomb collisions, $\rho_{e}$ is the gyroradius and $\delta$ is the characteristic size of the system. Let us examine these assumptions to determine if we can in fact expect the measured $\eta$ to follow the classical expression (1). For MRX the electron collision time is less then $10 \mathrm{~ns}$, and therefore the reconnection phase of the discharge provides sufficient time for a steady-state electron velocity distribution to be established. The electrons are strongly magnetized almost everywhere inside the current sheet: $\rho_{e} \sim 10^{-2} \mathrm{~cm} \ll \delta \sim 3 \mathrm{~cm}$, where $\delta$ is the thickness of the sheet; and $\rho_{e} \ll \lambda_{m f p}$, where the electron collision mean free path $\lambda_{m f p}$ varies between 0.1 and $100 \mathrm{~cm}$. Therefore, assumptions 1 and 3 are always satisfied in MRX. Depending on the operating conditions, assumptions 4-6 may or may not be satisfied; experimental data exhibiting distinct regimes will be presented below. 
The second condition is violated in the reconnection neutral sheet. The magnetic field in MRX has a null surface in the middle of the current sheet and, to the lowest order, varies linearly with radius away from null surface. The plasma and current density are both peaked at the magnetic null line, while Spitzer's resistivity calculations assume spatial uniformity. It is not obvious which value, $\eta_{\perp}$ or $\eta_{\|}$, if any, applies in the case of MRX current sheet. A detailed kinetic theory of resistivity in non-uniform magnetic field is beyond the scope of this letter. In the approximation of circular particle orbits, one can write the Fokker-Plank equation for the species $\alpha$ as

$$
\boldsymbol{v} \cdot \nabla f_{\alpha}-\Omega_{\alpha} \frac{\partial f_{\alpha}}{\partial \phi}+q_{\alpha} \boldsymbol{E} \cdot \frac{\partial f_{\alpha}}{\partial \boldsymbol{v}}=\left(\frac{\partial f_{\alpha}}{\partial t}\right)_{c}
$$

where $\alpha=i$ or $e, \phi$ is the gyrophase angle, $q_{\alpha}$ are the charges and $\Omega_{\alpha}=q_{\alpha} B / m$. $z$ is taken to be parallel to $B$, and all quantities are allowed to vary in the $x$ direction only. $(x$ corresponds to $R-R_{0}$ in the MRX device coordinates, where $R_{0}$ is the major radius of the field null). Then the diamagnetic current is in the $y$ direction. The right hand side of (2) is the collisional Fokker-Plank term. The zeroth order distribution functions are taken to be Maxwellian with densities $n_{\alpha}$ and temperatures $T_{\alpha}$ that depend on $x$ quadratically as

$$
\begin{aligned}
& n_{\alpha}=n_{\alpha 0}+n_{\alpha 0}^{\prime \prime} x^{2} / 2 \\
& T_{\alpha}=T_{\alpha 0}+T_{\alpha 0}^{\prime \prime} x^{2} / 2
\end{aligned}
$$

The distributions make the collisional terms and the terms containing $\Omega$ vanish. The magnetic field at the origin is linear so that $\Omega_{\alpha}=\Omega_{\alpha 0}^{\prime} x$. Using standard Fokker-Plank theory one finds the correction to the distribution function to the first order in both $1 /\left(\tau_{c} \Omega\right)$ and $e E \delta / T_{e}$, and by taking the first velocity moment of (2) the electron-ion friction force as

$$
R=n_{e} e \eta_{\perp}^{\text {Spitzer }} j-\frac{3}{2} n_{e} \eta_{\perp}^{\text {Spitzer }} e j_{e T}
$$

where $j_{e T} \equiv\left(\partial T_{e} / \partial x\right) /\left(e \eta_{\perp}^{\text {Spitzer }}\left|\Omega_{e}\right|\right)$ is the diamagnetic current resulting from the electron temperature gradient. The resulting resistivity is essentially equal to Spitzer's $\eta_{\perp}$ with a correction due to the temperature gradient. In MRX this correction is ignored since the 
measured radial temperature gradient is small and vanishes in the middle of the current sheet. Very close to $x=0$ the approximation on which the theory is based breaks down and the orbits change from circles to "figure eight" and betatron orbits. The distribution function for the betatron orbits is distorted as in Spitzer's $\eta_{\|}$calculation. However, only one half of the total number of electrons at $x=0$ are on these orbits. One could guess that here $\eta=2 \eta_{\|} \approx \eta_{\perp}$, so that there should be no major change in equation (3) even at $x=0$. The presence of field reversal therefore does not change the value of $\eta_{\perp}^{\text {Spitzer }}$. Let us now consider the experiment.

The present experiment relies on the diagnostics consisting of several arrays of internal magnetic pickup coil probes, and Langmuir probes. All three components of the magnetic field are measured by a 90-channel 2D pickup coil array. A finer 1D pickup coil array measures the $B_{z}$ profile across the current sheet with $5 \mathrm{~mm}$ resolution. The electron density and temperature are measured simultaneously by Langmuir probes with triple pins. Both the electric field and the plasma current density are determined from magnetic pickup coil probe data. The electric field $E_{T}$ is calculated from Faraday's law as $-(d \Psi / d t) / 2 \pi R$, where $\Psi(R, Z, t)$ is the poloidal magnetic flux. The poloidal flux function is determined experimentally as $\Psi(R, Z, t)=\int_{0}^{R} 2 \pi R^{\prime} B_{Z}\left(R^{\prime}, Z, t\right) d R^{\prime}$, where axisymmetry is assumed. The "pull" reconnection phase starts when an axially elongated 2-dimensional current sheet forms and persists for $\sim 20-30 \mu \mathrm{s}$. The current density is calculated from Ampere's law $j_{\theta}(R) \approx-\left(1 / \mu_{0}\right)\left(\partial B_{Z} / \partial R\right)$. Stable neutral current sheets are reproducibly obtained in MRX [6]. The radial profile of $B_{Z}$ was found to conform very closely to the hyperbolic tangent profile of the field in the Harris-type current layer [8]. Using this extremely robust result, we routinely determine the thickness of the current sheet $\delta$ as a fit parameter from the best fit of the experimental $B_{Z}$ profile to the hyperbolic tangent.

A typical MRX discharge has a time interval of approximately $20 \mu$ s duration that is characterized by a plateau of the total current and a spatially stationary neutral sheet. This is the portion of the discharge evolution we used for resistivity measurement. The toroidal component of the Ohm's law, $E_{T}+V_{R} B_{Z}=\eta j_{T}$ in the center of the current sheet reduces to 
simply $E_{T}=\eta j_{T}$. The electric field and current density are measured, and the expressions yields the resistivity that can be directly compared to the classical value.

The observed loop voltage, peak current density and resistivity are shown on Fig. 2 as functions of the fill pressure of hydrogen or deuterium gas. Also shown on Fig. 2 (c) with open symbols is the transverse Spitzer resistivity calculated using Eq. (1) based on measured $T_{e}$ and $n_{e}$. The measured resistivity is remarkably close to the classical value in the range of pressures above approximately 5 mTorr. Below 5 mTorr, the resistivity quickly becomes "anomalous". The normalized resistivity is plotted in Fig. 3 as a function of the collisionality parameter $\delta / \lambda_{\mathrm{mfp}}$. For the collision-dominated plasmas $\left(\delta / \lambda_{\mathrm{mfp}}>1\right)$ the resistivity is classical.

In our past papers, we stated that magnetic reconnection could not be generally explained by a classical theory based on collisions, and Fig. 3 proves this point once again. As the mean free path increases beyond the size of the plasma $\delta$, the resistivity is enhanced by up to an order of magnitude over the collisional value, indicating that dissipation mechanisms other than Coulomb collisions become dominant [9]. In particular, lower-hybrid drift turbulence has been observed in MRX current sheets [10], and this could contribute to the measured resistivity. However, a theoretical estimate of the effective collisionality, which is comparable to the lower hybrid frequency, shows that this contribution is insignificant in high collisionality discharges $\left(\nu_{e i} \gg f_{L H}\right)$.

The main claim of this letter is that we have found a regime in which the electronion Coulomb collisions play the main role in determining resistivity over other effects which includes Hall effects, turbulence and Pedersen currents. This regime is realized in MRX when the plasma density is high and the electron temperature is low, and the mean free path of electrons $(1-5 \mathrm{~mm})$ is much shorter than the shortest scale size of the neutral sheet $(1-2 \mathrm{~cm})$. In this limit, the collision rate greatly exceeds the characteristic turbulence frequencies and the resistive MHD Ohm's law is valid, and therefore, it can be used to measure resistivity.

A slight increase of the resistivity over the classical value is seen at pressures above $\sim 13$ mTorr (Fig. 2(c)). As the background neutral pressure increases, electron collisions 
with the background neutral gas atoms and molecules start to contribute to dissipation.

Based on the discharges that satisfy the high Coulomb collisionality criterion $\delta / \lambda_{\mathrm{mfp}}>$ 1 and are not neutral collision dominated (fill pressure under 13 mTorr), the transverse resistivity was found to be $\eta_{\perp}=(1.1 \pm 0.09) \eta_{\perp}^{\text {Spitzer }}$. The error estimate includes only statistical uncertainty due shot-to-shot variations and temporal variations of measured values during each shot. In order to verify the $T_{e}$ measurement by the triple Langmuir probe we compared it with $T_{e}$ measured independently from (1) $I-V$ curve measured by scanning the bias voltage of a double probe at the conditions of the present experiments and (2) spectroscopic measurement using He I line intensity ratio method and collisional-radiative model data of Sasaki et al [7] for helium plasmas with $T_{e}$ in the 10 to $20 \mathrm{eV}$ range. Based on these comparisons, we estimate that the systematic error of the $T_{e}$ measurement is in the 10 to $20 \%$ range. This results in the total uncertainty of approximately $30 \%$ for the normalized resistivity $\eta_{\perp} / \eta_{\perp}^{\text {Spitzer }}$

To verify the electron temperature scaling behavior of the resistivity in the collisional regime, a series of shots were made at fixed pressure of 6 mTorr. The capacitor bank voltage, and therefore the energy deposited into the plasma was varied in order to obtain a range of $T_{e}$ values. The resulting temperature dependence (Fig. 4) based on the shots satisfying the high collisionality condition $\delta / \lambda_{\operatorname{mfp}}>1$ agrees with the $T_{e}^{-3 / 2}$ scaling of the Spitzer formula. In order to separate the $T_{e}$ and density dependence, Eq. (1) can be rearranged as follows:

$$
9.71 \times 10^{3} \eta T_{e}^{3 / 2}-(3 / 2) \ln \left(T_{e}\right)=23-(1 / 2) \ln \left(n_{e}\right)
$$

where $T_{e}$ is in $\mathrm{eV}$ and $n_{e}$ is in $\mathrm{cm}^{-3}$, and an approximate expression for $\ln \Lambda$ was used. The left hand side of (4) was evaluated using experimental data and plotted as a function of density in Fig. 5 (circles). The right hand side (dotted line) is a function of density only. The weak density dependence is consistent with the logarithmic scaling of the Spitzer formula across the entire one order of magnitude range.

In summary, the transverse resistivity of a plasma with singly ionized ions has been measured over a range of collisionality conditions. The assumptions of the Spitzer model hold 
for the steady-state phase of MRX discharges with the collisionality parameter $\delta / \lambda_{\mathrm{mfp}} \gg 1$. In the parameter range where this condition is satisfied, the resistivity was found to agree to the calculated Spitzer value to within 30\% uncertainty. To our knowledge, this is the most accurate measurement of the transverse Spitzer resistivity to date. The observed resistivity follows the $T_{e}^{-3 / 2}$ dependence characteristic of Coulomb collisions, and shows no significant $n_{e}$ dependence. A transition from Coulomb to anomalous dissipation is observed as collisionality is decreased. Evidence of the contribution of electron-neutral collisions to the resistivity is observed at high fill pressures.

The authors are thankful to R. Cutler and D. Cylinder for excellent technical support and to Y. Ren for help with operation of the experiment. This work was supported by the NSF, NASA and the U.S. DOE Contract No. AC02-76CH0-3073. 


\section{REFERENCES}

* Present address: Cymer, Inc., San Diego, CA. Electronic mail address: fedor@pppl.gov

[1] L. Spitzer, Physics of fully ionized gases. (Interscience Publishers, New York, 1956)

[2] N. Rynn, Phys. Fluids, 7, 284, (1964).

[3] N. Bretz, D.L. Dimock, E. Hinnov, and E.B. Meservey, Nucl. Fusion, 15, 313 (1975).

[4] For example, R.J. Commisso and H.R. Griem, Phys. Fluids, 20, 44 (1977).

[5] For a review of FRC experiments see M. Tuszewski, Nucl. Fusion, 28, 2033 (1988).

[6] M. Yamada et al., Phys. Plasmas, 4, 1936 (1997).

[7] S. Sasaki et al., National Institute for Fusion Science research report 346, 1995.

[8] M. Yamada et al., Phys. Plasmas, 7, 1781 (2000).

[9] H. Ji, M. Yamada, S. Hsu, and R. Kulsrud, Phys. Rev. Lett., 80, 3256, (1998).

[10] T. A. Carter, H. Ji, F. Trintchouk, M. Yamada, and R. M. Kulsrud, Phys. Rev. Lett., 88, 015001, (2001). 


\section{FIGURES}

(a)

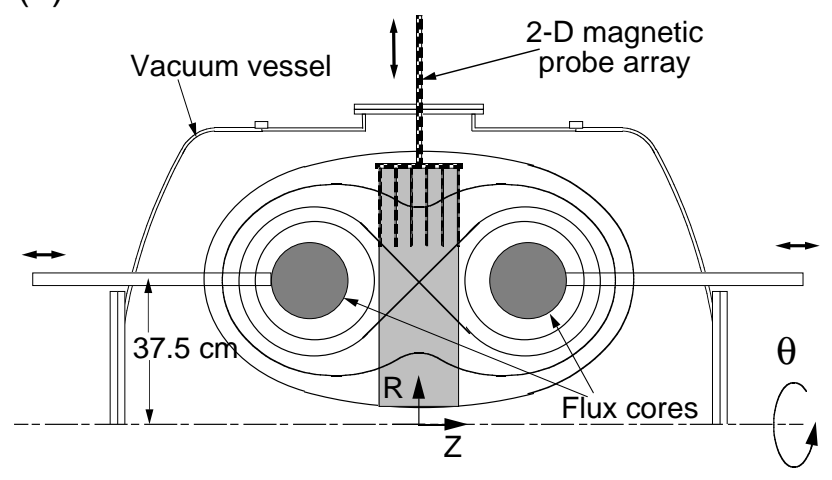

(b)

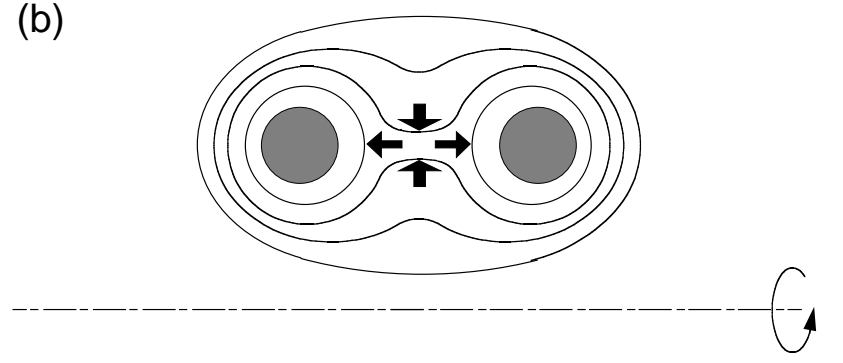

FIG. 1. MRX apparatus and magnetic field geometry 


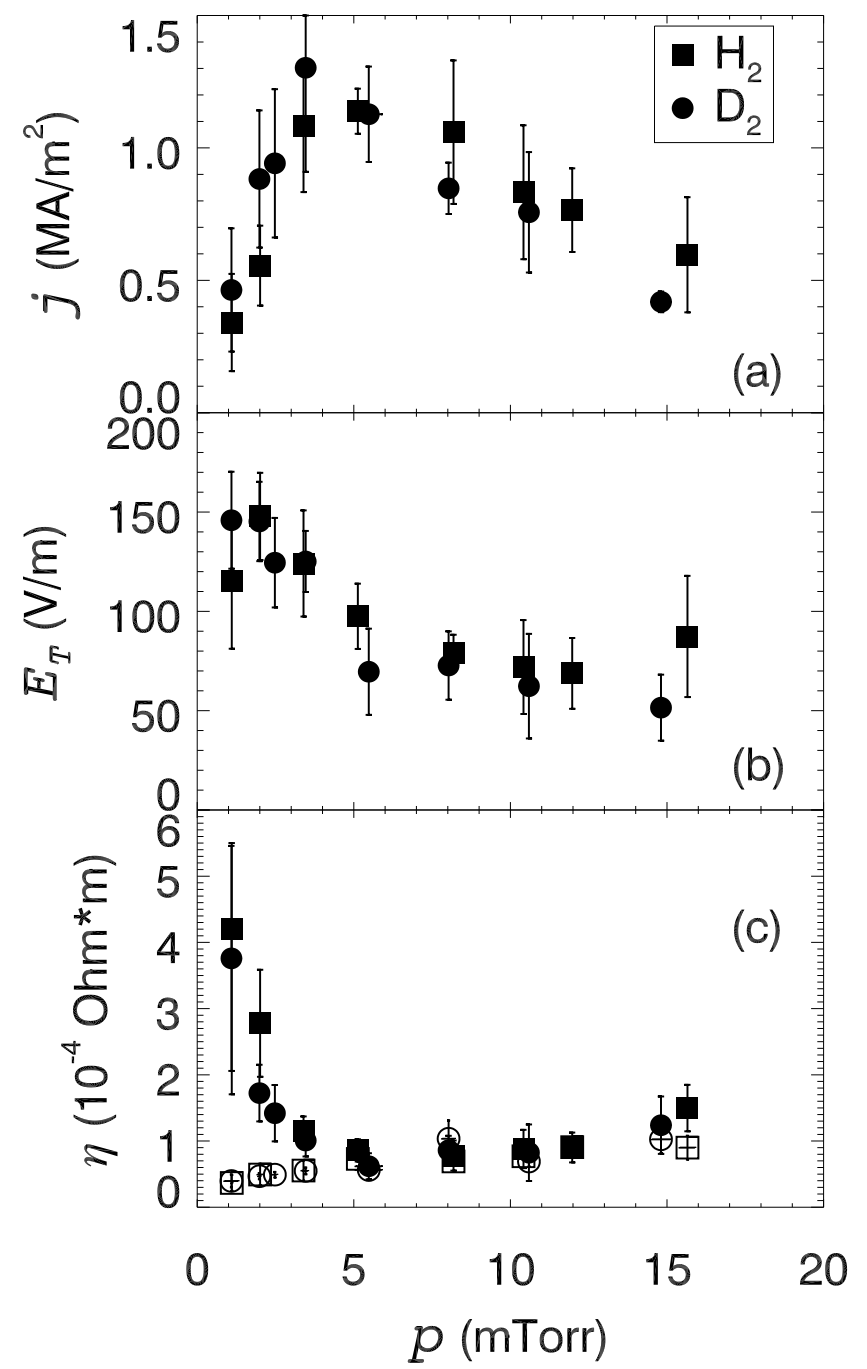

FIG. 2. Toroidal current density(a), electric field (b), and resistivity (c) vs. neutral fill pressure for $\mathrm{H}_{2}$ (squares) and $\mathrm{D}_{2}$ (circles). Open symbols in (c) correspond to $\eta_{\perp}^{\text {Spitzer }}$, filled symbols measured resistivity 


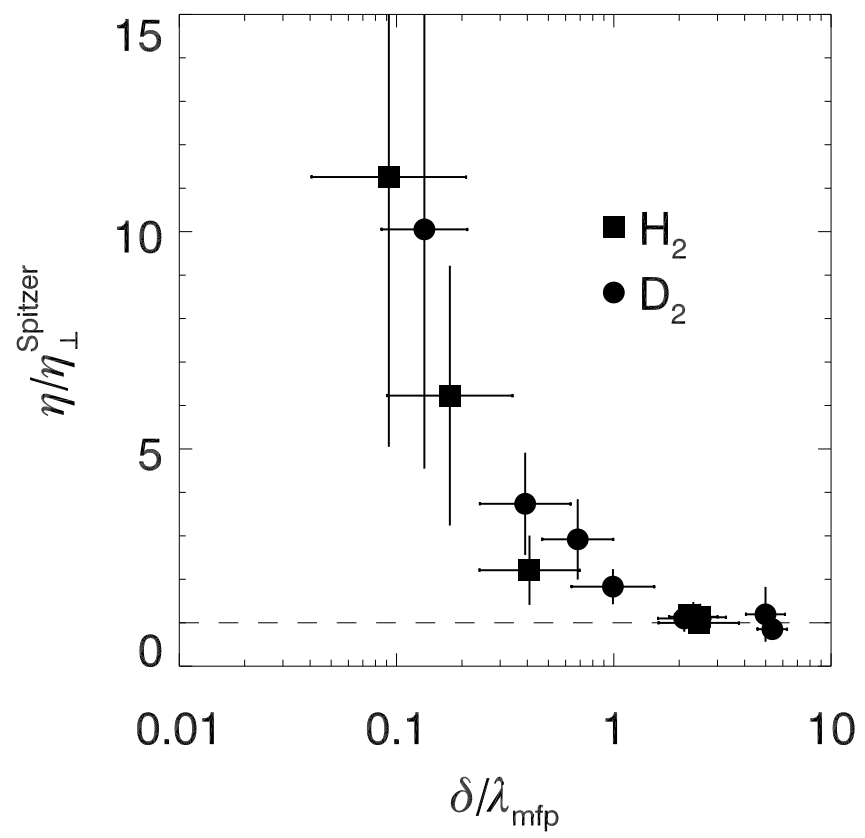

FIG. 3. Resistivity anomaly factor $\eta / \eta_{\perp}^{\text {Spitzer }}$ as a function of the collisionality parameter $\delta / \lambda_{\mathrm{mfp}} \cdot p<13$ mTorr.

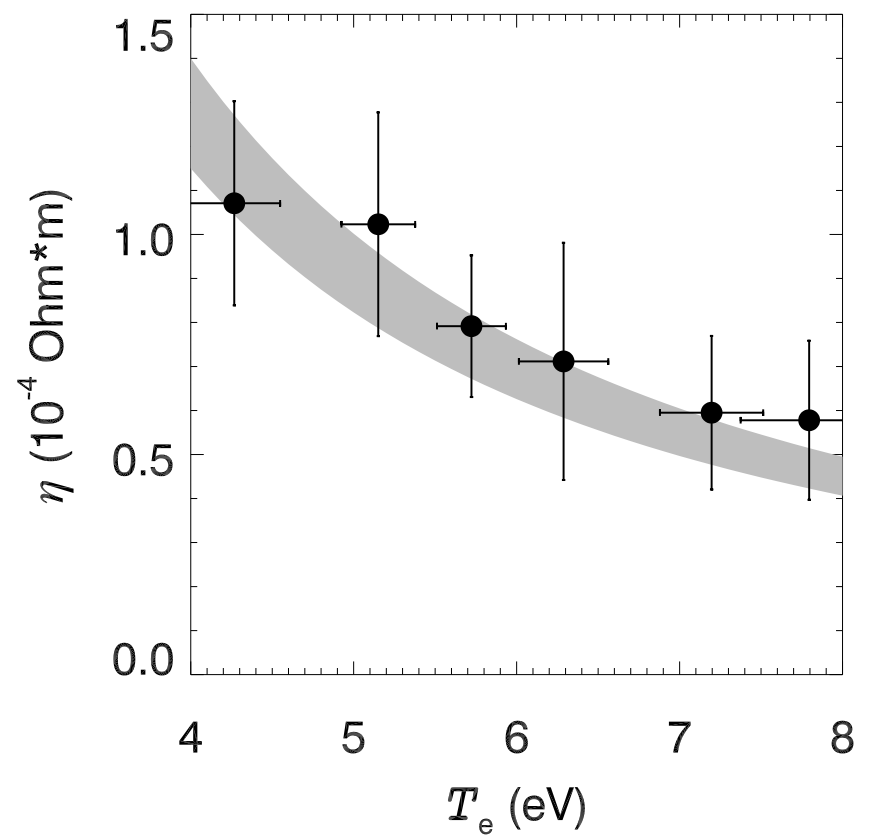

FIG. 4. $T_{e}$ dependence of the measured resistivity (open circles) and $\eta_{\perp}^{\text {Spitzer }}$ calculated from measured $T_{e}$ and $n_{e}$ (shaded area). The one- $\sigma$ range of $\eta_{\perp}^{\text {Spitzer }}$ values shown incorporates the statistical uncertainty of $T_{e}$ measurement and the $n_{e}$ variations in the data set. Fill pressure $p=6$ mTorr. 


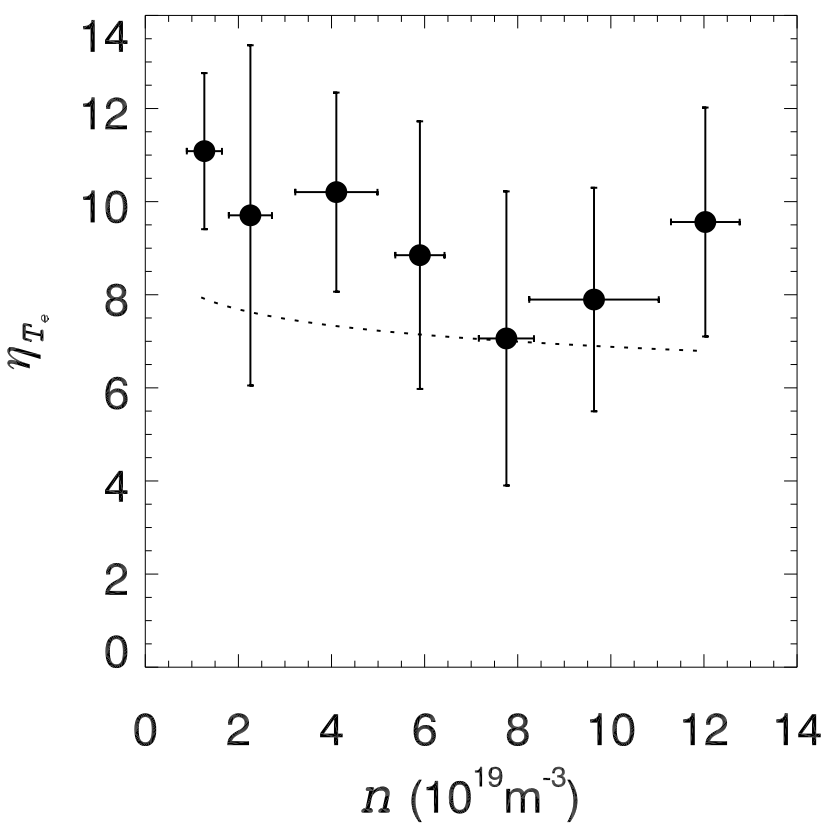

FIG. 5. Filled circles: measured values of the temperature-normalized resistivity $\eta_{T_{e}} \equiv 9.71 \times 10^{3} \eta T_{e}^{3 / 2}-(3 / 2) \ln \left(T_{e}\right)$ (left hand side of $\left.(4)\right)$; dotted line: $23-(1 / 2) \ln \left(n_{e}\right)$ vs. electron density. 


\section{External Distribution}

Plasma Research Laboratory, Australian National University, Australia

Professor I.R. J ones, Flinders University, Australia

Professor J oão Canalle, Instituto de Fisica DEQ/IF - UERJ , Brazil

Mr. Gerson O. Ludwig, Instituto Nacional de Pesquisas, Brazil

Dr. P.H. Sakanaka, Instituto Fisica, Brazil

The Librarian, Culham Laboratory, England

Library, R61, Rutherford Appleton Laboratory, England

Mrs. S.A. Hutchinson, JET Library, England

Professor M.N. Bussac, Ecole Polytechnique, France

Librarian, Max-Planck-Institut für Plasmaphysik, Germany

J olan Moldvai, Reports Library, MTA KFKI-ATKI, Hungary

Dr. P. Kaw, Institute for Plasma Research, India

Ms. P.J . Pathak, Librarian, Insitute for Plasma Research, India

Ms. Clelia De Palo, Associazione EURATOM-ENEA, I taly

Dr. G. Grosso, Instituto di Fisica del Plasma, Italy

Librarian, Naka Fusion Research Establishment, J AERI, J apan

Library, Plasma Physics Laboratory, Kyoto University, J apan

Research Information Center, National Institute for Fusion Science, J apan

Dr. O. Mitarai, Kyushu Tokai University, J apan

Library, Academia Sinica, Institute of Plasma Physics, People's Republic of China

Shih-Tung Tsai, Institute of Physics, Chinese Academy of Sciences, People's Republic of China

Dr. S. Mirnov, TRINITI, Troitsk, Russian Federation, Russia

Dr. V.S. Strelkov, Kurchatov Institute, Russian Federation, Russia

Professor Peter Lukac, Katedra Fyziky Plazmy MFF UK, Mlynska dolina F-2, Komenskeho Univerzita, SK-842 15 Bratislava, Slovakia

Dr. G.S. Lee, Korea Basic Science Institute, South Korea

Mr. Dennis Bruggink, Fusion Library, University of Wisconsin, USA

Institute for Plasma Research, University of Maryland, USA

Librarian, Fusion Energy Division, Oak Ridge National Laboratory, USA

Librarian, Institute of Fusion Studies, University of Texas, USA

Librarian, Magnetic Fusion Program, Lawrence Livermore National Laboratory, USA

Library, General Atomics, USA

Plasma Physics Group, Fusion Energy Research Program, University of California at San Diego, USA

Plasma Physics Library, Columbia University, USA

Alkesh Punjabi, Center for Fusion Research and Training, Hampton University, USA

Dr. W.M. Stacey, Fusion Research Center, Georgia Institute of Technology, USA

Dr. J ohn Willis, U.S. Department of Energy, Office of Fusion Energy Sciences, USA

Mr. Paul H. Wright, Indianapolis, Indiana, USA 
The Princeton Plasma Physics Laboratory is operated by Princeton University under contract with the U.S. Department of Energy.

\author{
Information Services \\ Princeton Plasma Physics Laboratory \\ P.O. Box 451 \\ Princeton, NJ 08543
}

Phone: 609-243-2750

Fax: 609-243-2751

e-mail: pppl_info@pppl.gov

Internet Address: http://www.pppl.gov 